\title{
APPROXIMATION PROBLEMS IN MODULAR SPACES OF DOUBLE SEQUENCES
}

\author{
ALEKSANDER WASZAK
}

\begin{abstract}
Let $X$ denote the space of all real, bounded double sequences, and let $\Phi, \varphi, \Gamma$ be $\varphi$-functions. Moreover, let $\Psi$ be an increasing, continuous function for $u \geq 0$ such that $\Psi(0)=0$.

In this paper we consider some spaces of double sequences provided with two-modular structure given by generalized variations and the transiation operator

We apply the $\gamma\left(\tilde{v}_{\Phi}, \tilde{\rho}_{\varphi}\right)$-convergence in $\bar{X}(\Phi, \Psi)$ in order to oblain an approximation theorem by means of the $(m, n)$-transiation, i.e. a result of the form $\left(\tau_{m n} z-x\right) \rightarrow 0$ in an Orlicz sequence space $l^{\Gamma}$.
\end{abstract}

\section{Notation}

1.1. A function $\varphi$ defined in the interval $[0, \infty)$, continuous and nondecreasing for $u \geq 0$ and such that $\varphi(u)>0$ for $u>0, \varphi(u) \rightarrow \infty$ as $u \rightarrow \infty$ and $\varphi(0)=0$, is called a $\varphi$-function. We will consider three $\varphi$-functions $\Phi, \varphi$ and $\Gamma$. Moreover, let $\Psi$ be a nonnegative, nondecreasing function of $u \geq 0$ such that $\Psi(u) \rightarrow 0$ as $u \rightarrow 0+$, , (see [3]).

1.2. Let $X$ be the space of all real, bounded double sequences. Throughout this paper sequences belonging to $X$ will be denoted by $x=\left(t_{\mu \nu}\right)=\left((x)_{\mu \nu}\right)$ or $\left(t_{\mu \nu}\right)_{\mu, \nu=0}^{\infty}=\left((x\rangle_{\mu \nu}\right)_{\mu, \nu=0}^{\infty}$ and $|x|=\left(\left|t_{\mu \nu}\right|\right), y=\left(s_{\mu \nu}\right), x^{p}\left(t_{\mu \nu}^{p}\right)$ for $p=1,2, \ldots$ By a convergent sequence we shall mean a double sequence converging in the sense of Pringsheim. The symbols $X_{d}$ or $X_{1}$ denote subspaces of the space $X$ such that, for every fixed $\bar{\mu}$ and for every fixed $\bar{\nu}$ the sequences $\left(t_{\bar{\mu} \nu}\right)$ and $\left(t_{\mu \bar{\nu}}\right)$ are nonincreasing or nondecreasing, respectively.

1.3. Let $\rho_{\varphi}: X \rightarrow(0, \infty)$ be a functional generated by the $\varphi$-function $\varphi$ such that for arbitrary $x, y \in X$ and $\alpha, \beta \geq 0$.

$$
\begin{aligned}
& \text { 1' } \rho_{\varphi}(0)=0, \\
& 1^{\prime \prime} \rho_{\varphi}(x)=0 \text { implics } x=0, \\
& \text { 2' } \rho_{\varphi}(-x)=\rho_{\varphi}(x), \\
& \text { 3' } \rho_{\varphi}(\alpha x+\beta y) \leq \rho_{\varphi}(x)+\rho_{\varphi}(y) \text {, for } \alpha+\beta=1,
\end{aligned}
$$




\section{Completeness of a two-modular space}

3.1. We are now going to investigate the completeness of two-modular space $\left\langle\hat{X}(\Phi, \Psi), \tilde{v}_{\Phi}, \tilde{\rho}_{\varphi}\right)$. The theorems on completeness of the spaces $\tilde{X}_{\rho_{\varphi}}$ and $\tilde{X}_{\varphi}(\Psi)$ with respect to the $F$-norm $\|\cdot\|_{\rho_{\varphi}}$ or the modular functional $\tilde{\rho}_{\varphi}$ have been obtained in [7] (compare also [5]). Let us remark that the space $\tilde{X}(\Phi, \Psi)$ is not complete with respect to $\|\cdot\|_{p_{\varphi}}$ and $\tilde{\rho}_{\varphi}$, respectively. Indeed, consider the following example.

Let $\Phi(u)=|u|, \varphi(u)=|u|, \Psi(u)=u^{2}$ and $x=\left(t_{\mu \nu}\right)_{\mu, \nu=0}^{\infty}, x^{p}=\left(t_{\mu \nu}^{p}\right)_{p, \nu=0^{x}}^{\infty}$ $p=1,2, \ldots$, where

$$
t_{\mu \nu}=\left\{\begin{array}{ll}
\frac{1}{(\mu+1)(\nu+1)} & \text { for } \mu=\nu, \\
0 & \text { elsewhere }
\end{array} \quad t_{\mu \nu}^{p}= \begin{cases}t_{\mu \nu} & \text { for } \mu \leq p \text { and } \nu \leq p, \\
0 & \text { elsewhere. }\end{cases}\right.
$$

Since

$$
\begin{aligned}
\omega_{\varphi}\left(x^{p} ; r, s\right) \leq \sup _{m \geq r \geq p} \sup _{p \geq p \geq m p \geq \nu \geq n} \sup _{p} \frac{2}{(\mu+1)(\nu+1)} \leq \frac{2}{(r+1)(s+1)}, \\
r s \Psi\left(\omega_{\varphi}\left(x^{p} ; r, s\right)\right) \leq \frac{4}{(r+1)(s+1)} \rightarrow 0 \text { as } r, s \rightarrow \infty
\end{aligned}
$$

and

$$
v_{\Phi}\left(x^{p}\right)=\sum_{1 \leq \mu, \nu \leq p}\left(t_{\mu, \nu}+t_{\mu-1, \nu-1}\right)=1+\frac{1}{(p+1)^{2}}+2 \sum_{\mu=1}^{p-1} \frac{1}{(\mu+1)^{2}}<\infty
$$

then $x^{p} \in X(\Phi, \Psi)$. Further, if $r<p$ and $s<p$, we have

$$
\omega_{\varphi}\left(x^{p}-x ; r, s\right) \leq \frac{2}{(p+1)^{2}}, r s \Psi\left(\omega_{\varphi}\left(x^{p}-x ; r, s\right)\right) \leq \frac{4}{(p+1)^{2}},
$$

if $r \geq p$ and $s \geq p$, we have,

$$
\begin{aligned}
\omega_{\varphi}\left(x^{p}-x ; r, s\right) \leq \frac{2}{(r+1)(s+1)}, r s \Psi\left(\omega_{\varphi}\left(x^{p}-x ; r, s\right)\right) & \leq \\
& \leq \frac{4}{(r+1)(s+1)} \leq \frac{4}{(p+1)^{2}}
\end{aligned}
$$

and in consequence we obtain

$$
\rho_{\varphi}\left(x^{p}-x\right)=\sup _{r, g} r s\left(\omega_{\varphi}\left(x^{p}-x ; r, s\right)\right) \leq \frac{4}{(p+1)^{2}} \rightarrow 0 \text { as } p \rightarrow \infty \text {. }
$$

This shows that $x^{p} \rightarrow x$ in the $F$-norm of $X_{\varphi}(\Psi)$. Moreover, we have

$$
r s \Psi\left(\omega_{\varphi}(x ; r, s)\right) \leq \frac{4}{(r+1)(s+1)} \rightarrow 0 \text { as } r, s \rightarrow \infty,
$$

and so $x \in X_{\varphi}(\Psi)$. However

$$
\text { : } \quad v_{\phi}(x)=\sum_{\mu, \nu=1}^{\infty}\left|t_{\mu, \nu}+t_{\mu-1, \nu-1}\right| \geq 2 \sum_{\mu, \nu=1}^{\infty} \frac{1}{(\mu+1)(\nu+1)}=\infty,
$$

whence $x \notin X_{\Phi}$. Finally $x^{p} \in X(\Phi, \Psi), \rho_{\varphi}\left(x^{p}-x\right) \rightarrow 0$ as $p \rightarrow \infty$, but $x \notin X(\Phi, \Psi)$. 
3.2. In the sequel, for a given sequence $x \in X$ we define a new sequence $\bar{x}=\left(\bar{t}_{\mu \nu}\right)_{\mu \nu=0}^{\infty}$ by the formulas

$$
\vec{t}_{\mu \nu}= \begin{cases}t_{\mu 0}+a, & \text { for } \mu=0,1,2, \ldots \text { and } \nu=0 \\ t_{0 \nu}+a, & \text { for } \mu=0 \text { and } \nu=1,2, \ldots, \\ t_{\mu \nu}+b, & \text { for } \mu \geq 1 \text { and } \nu \geq 1\end{cases}
$$

where the constants $a$ and $b$ can be of the form $a=t_{\mu \nu}-t_{\mu 0}, b=t_{0 \nu}-t_{00}$ ( $\mu, \nu>0$ are arbitrary indices). In the following we shall consider the sequence $\bar{x}$ defined by the constants $a=t_{11}-t_{10}$ and $b=t_{01}-t_{00}$.

Remark. The following identity holds $\tilde{v}_{\Phi}(\tilde{x})=v_{\Phi}(\bar{x})$.

Proof: Since $\bar{x} \in \tilde{x}$, then by definition of $\tilde{v}_{\Phi}(\tilde{x})$ we have

$$
\tilde{v}_{\Phi}(\tilde{x}) \leq v_{\Phi}(\bar{x}) .
$$

Now, let $y=\left(s_{\mu \nu}\right)_{\mu, \nu=0}^{\infty} \in \tilde{x}$, then $s_{\mu 0}=t_{\mu 0}+A, s_{0 \nu}=t_{0 \nu}+A$ for $\mu=$ $0,1,2, \ldots, \nu=1,2, \ldots$ and $s_{\mu \nu}=t_{\mu \nu}+B$ for $\mu \geq 1$ and $\nu \geq 1$, where $A$ and $B$ are two arbitrary numbers. In the following we may define the sequence $\bar{y}=\left(\bar{s}_{\mu \nu}\right)_{\mu, \nu=0}^{\infty}$, where $\bar{s}_{\mu 0}=t_{\mu 0}+A+\alpha$, for $\mu=0,1,2, \ldots, \bar{s}_{0 \nu}=t_{0 \nu}+A+a$, for $\nu=1,2, \ldots$, and $\bar{s}_{\mu \nu}=t_{\mu \nu}+B+b$ for $\mu \geq 1$ and $\nu \geq 1$, with $a=t_{11}+B-t_{10}-A$ and $b=t_{01}=t_{00}$. Obviously, $v_{\Phi}(y) \geq v_{\Phi}(\bar{y})$ and $v_{\Phi}(\bar{y})=v_{\Phi}(\bar{x})$. Hence, $v_{\Phi}(y) \geq v_{\Phi}(\bar{x})$ for every $y \in \tilde{x}$. In consequence

$$
\tilde{v}_{\Phi}(\tilde{x}) \geq v_{\Phi}(\bar{x}) \text {. }
$$

Finally, by $(t)$ and $(t+)$ we obtain $\tilde{v}_{\Phi}(\tilde{x})=v_{\Phi}(\bar{x})$.

3.3. Theorem. Let $\Phi, \varphi$ be $\varphi$-functions and let $\Psi$ be the function defined as in 1.1., which satisifies the condition:

there exists a $u_{0}>0$ such that for every $\delta>0$ there is an $\eta>0$ satisfying the inequality $\Psi(\eta u) \leq \delta \Psi(u)$ for all $0 \leq u \leq u_{0}$.

Then, the two-modular space $\left\langle\tilde{X}(\Phi, \Psi), \tilde{v}_{\Phi}, \tilde{\rho}_{\varphi}\right\rangle$ is $\gamma$-complete.

Proof: Let us suppose that $\tilde{K}$ is a $\tilde{v}_{\Phi}$-ball in $\tilde{X}(\Phi, \Psi)$ and let $\tilde{x}^{p} \in \tilde{K}$ for $p=1,2, \ldots,\left(\tilde{x}^{p}\right)$ be a $\tilde{\rho}_{\varphi}$-Cauchy sequence. It is easily seen that the sequence $\left(\tilde{x}^{p}\right)$ is $\tilde{\rho}_{\varphi}$-convergent to an element $\tilde{x} \in \tilde{X}_{\varphi}(\Psi)$, (see [7] or compare [5]). In consequence $\tilde{x}^{p} \stackrel{\gamma}{\rightarrow} x$, where $\gamma=\gamma\left(\tilde{v}_{\Phi}, \tilde{\rho}_{\varphi}\right)$. Next, we show that $\tilde{x} \in \tilde{K}$. Taking the sequence $\left(x^{p}\right)$, such that $x^{p} \in \tilde{x}^{p}, x^{p} \in X_{\Phi}$ we may define the sequence $\left(\bar{x}^{p}\right)$. Of course, we have

$$
v_{\Phi}\left(k_{0} \bar{x}^{p}\right) \leq M_{0}
$$

for some positive numbers $k_{0}$ and $M_{0}$. If $\bar{x}^{p}=\left(\bar{t}_{\mu \nu}^{p}\right)$, then

$$
\sum_{\mu, \nu=1}^{\infty} \Phi\left(k_{0}\left|\bar{t}_{m_{\mu-1}, n_{\nu}-1}^{p}-\bar{t}_{m_{\mu-1}, n_{\nu}}^{p}-\bar{t}_{m_{\nu}, n_{\nu-1}}^{P}+\bar{t}_{m_{\mu}, n_{\nu}}^{p}\right|\right) \leq M_{0}
$$


for all increasing sequences $\left(m_{\mu}\right)$ and $\left(n_{\nu}\right)$ of positive integers and for $p=$ $1,2, \ldots$. Since $\bar{t}_{\mu \nu}^{p} \rightarrow \bar{t}_{\mu \nu}$ as $p \rightarrow \infty$ for every $\mu$ and $\nu$, where $\left(\bar{t}_{\mu \nu}\right)=\bar{x}$, then we easily obtain

$$
\sum_{\mu, \nu=1}^{\infty} \Phi\left(k_{0}\left|\bar{t}_{m_{\mu-1}, n_{\nu-1}}-\bar{t}_{m_{\mu-1}, n_{\nu}}-\bar{t}_{m_{\mu}, n_{\nu-1}}+\bar{t}_{m_{\mu}, n_{\nu}}\right|\right) \leq M_{0}
$$

for $\left(m_{\mu}\right),\left(n_{\nu}\right), p$ as previously. Therefore $v_{\Phi}\left(k_{0} \bar{x}\right) \leq M_{0}$. Applying the above remark, we obtain $\tilde{v}_{\Phi}\left(k_{0} \tilde{x}\right) \leq M_{0}$, and consequently $\tilde{x} \in \tilde{K}$.

\section{A theorem of approximation type}

4.1. Let $\Phi, \varphi, \Psi, \Gamma$ be the functions defined as in part 1.1. We shall consider an Orlicz sequence space $l^{\Gamma}$ and the space $\tilde{X}(\Phi, \Psi)$, and we shall apply the $\gamma$ convergence in $\dot{X}(\Phi, \Psi)$ in order to formulate a theorem of the form $\tau_{m n} x-x \rightarrow$ 0 in the space $i^{r}$.

Let us denote $T(x, m, n, \mu, \nu)=\left|\left(\tau_{m n} x\right)_{\mu \nu}-(x)_{\mu \nu}\right|$ and $M(x, m, n, \mu, \nu)=$ $\left|t_{\mu+m, \nu+n}-t_{\mu+m, \nu}-t_{\mu, \nu+n}+t_{\mu, \nu}\right|$, for all $m, n, \mu, \nu$.

\section{Lemma.}

(a) If $x \in X_{d}$, then $T(x, m, n, \mu, \nu) \leq M(x, m, n, \mu, \nu)$ for all $m, n, \mu$ and $\nu$.

(b) If $x \in X_{i}$, then $T(x, m, n, \mu, \nu) \leq M(x, m, n, \mu, \nu)$ for all $m, n, \mu$ and $\nu$.

Proof (a): For $\mu<m$ and $\nu<n$ we have $T(x, m, n, \mu, \nu)=0$.

If $\mu \geq m$ and $\nu<n$, then $T(x, m, n, \mu, \nu)=\left|t_{\mu+m, \nu}-t_{\mu, \nu}\right| \leq \mid\left(t_{\mu, \nu+n}-\right.$ $\left.t_{\mu+m, \nu+n}\right)+\left(t_{\mu+m, \nu}-t_{\mu, \nu}\right) \mid=M(x, m, n, \mu, \nu)$.

If $\mu<m$ and $\nu \geq n$, then $T(x, m, n, \mu, \nu)=\left|t_{\mu, \nu+n}-t_{\mu, \nu}\right| \leq \mid\left(t_{\mu+m, \nu}-\right.$ $\left.t_{\mu+m, \nu+n}\right)+\left(t_{\mu, \nu+n}-t_{\mu, \nu}\right) \mid=M(x, m, n, \mu, \nu)$.

For $\mu \geq m$ and $\nu \geq n$ we have $T(x, m, n, \mu, \nu)=\left|t_{\mu+m, \nu+n}-t_{\mu, \nu}\right| \leq$ $\mid\left(t_{\mu+m, \nu+n}-t_{\beta, \nu}\right)+\left(t_{k, \nu}-t_{\mu+m, \nu}\right)+\left(t_{\mu, \nu}-t_{\mu, \nu+n}\right)=M(x, m, n, \mu, \nu)$.

Finally $T(x, m, n, \mu, \nu) \leq M(x, m, n, \mu, \nu)$ for all $m, n, \mu$ and $\nu$.

Proof (b): For $\mu<m$ and $\nu<n,\left(\tau_{m n} x\right)_{\mu \nu}=t_{\mu \nu}$, then $T(x, m, n, \mu, \nu)=0$.

If $\mu \geq m$ and $\nu<n$, then $T(x, m, n, \mu, \nu)=\left|t_{\mu+m, \nu}-t_{\mu, \nu}\right| \leq \mid\left(t_{\mu, \nu}-t_{\mu+m, \nu}\right)+$ $\left(t_{\mu+m, \nu+n}-t_{\mu, \nu+n}\right) \mid=M(x, m, n, \mu, \nu)$.

If $\mu<m$ and $\nu \geq n$, then $T(x, m, n, \mu, \nu)=\left|t_{\mu, \nu+n}-t_{\mu, \nu}\right| \leq \mid\left(t_{\mu, \nu}-t_{\mu, \nu+n}\right)+$ $\left(t_{\mu+m, \nu+n}-t_{\mu+m, \nu}\right) \mid=M(x, m, n, \mu, \nu)$.

For $\mu \geq m$ and $\nu \geq n$ we have $T(x, m, n, \mu, \nu)=\left|t_{\mu+m, \nu+n}-t_{\mu, \nu}\right| \leq \mid\left(t_{\mu, \nu}-\right.$ $\left.t_{\mu+m, \nu+n}\right)+\left(t_{\mu, \nu+n}-t_{\mu, \nu}\right)+\left(t_{\mu+m, \nu}-t_{\mu, \nu}\right) \mid=M(x, m, n, \mu, \nu)$.

Thus $T(x, m, n, \mu, \nu) \leq M(x, m, n, \mu, \nu)$ for all $m, n, \mu$ and $\nu$. 
4.2. Let us suppose that the functions $\Phi, \varphi, \Gamma$ and $\Psi$ satisfy the following condition:

(i) There exist positive constants $a, b, u_{0}$ such that

$$
\Gamma(a u) \leq b \Phi(u) \Psi(\varphi(u)) \text { for } 0 \leq u \leq u_{0} \text {. }
$$

First let us remark that the condition (i) is equivalent to the following one:

(ii) For every $u_{1} \geq 0$ there exists a constant $c>0$ such that

$$
\Gamma(c u) \leq b \Phi(u) \Psi(\varphi(u)) \text { for } 0 \leq u \leq u_{1} \text {, (for a proof see [5]). }
$$

4.3. Let the functions $\Phi, \varphi, \Psi, \Gamma$ satisfy the assumptions 1.1 . and 4.2 , and let $v_{\Phi}(\lambda x)<\infty$ for a $\lambda>0$.

Theorem 1. If $x \in X_{d}$ or $x \in X_{i}$, then

$$
\sum_{\mu, \nu}^{\infty} \Gamma\left(c \lambda\left|\left(\tau_{r}, x\right)_{\mu \nu}-(x)_{\mu \nu}\right|\right) \leq b r s \Psi\left(\omega_{\varphi}(\lambda x ; r, s)\right) v_{\Phi}(\lambda x)
$$

for all nonnegative integers $r$ and $s$, where $c$ and b are some positive constants.

Proof: We limit ourselves to the case when $x \in X_{d}$. By Lemma we have $\left|\left(\tau_{m n} x\right)_{\mu \nu}-(x)_{\mu \nu}\right| \leq\left|t_{\mu, \nu}-t_{\mu+m, \nu}-t_{\mu, \nu+n}+t_{\mu+m, \nu+n}\right|$ for arbitrary $m, n, \mu$ and $\nu$. Let a positive constant $\lambda$ and integers $r$ and $s$ be given. Since $x$ is a bounded sequence, taking $u_{1}=4 \lambda \sup _{\mu, \nu}\left|t_{\mu, \nu}\right|$, and choosing $m \geq r, n \geq s$ arbitrary, by (i) we obtain

$$
\Gamma(c \lambda M(x, m, n, \mu, \nu)) \leq b \Phi(\lambda M(x, m, n, \mu, \nu)) \Psi(\varphi(\lambda M(x, m, n, \mu, \nu)))
$$

for all $m, n, \mu, \nu$ such that $\lambda M(x, m, n, \mu, \nu) \leq u_{1}$. We have

$$
\begin{aligned}
& \sum_{\mu, \nu=0}^{\infty} \Gamma\left(c \lambda\left|\left(\tau_{m n} x\right)_{\mu \nu}-(x)_{\mu \nu}\right|\right) \leq \\
& \leq b \Psi\left(\sup _{m \geq r \geq s} \sup _{n \geq \beta \geq m \geq n} \sup _{\nu \geq n} \varphi(\lambda M(x, m, n, \mu, \nu))\right) \sum_{\mu \geq m, \nu \geq n} \Phi(\lambda M(x, m, n, \mu, \nu))= \\
& =b \Psi\left(\omega_{\varphi}(\lambda x ; r, s)\right) \sum_{k, l=1}^{\infty} \sum_{\mu=k m}^{(k+1) m-1} \sum_{\nu=l n}^{(l+1) n-1} \Phi(\lambda M(x, m, n, \mu, \nu))= \\
& =b \Psi\left(\omega_{\varphi}(\lambda 2 ; r, s)\right) \sum_{k, i=1}^{\infty} \sum_{u=m}^{2 m-1} \sum_{v=n}^{2 n-1} \Phi\left(\lambda \mid t_{k m+u, l n+v}-t_{k m+u,(l-1) n+v}-\right. \\
& \left.-t_{(k-1) m+u, i n+v}+t_{(k-1) m+u,(1-1) n+v} \mid\right)= \\
& =b \Psi\left(\omega_{\varphi}(\lambda x ; r, s)\right) \sum_{u=m}^{2 m-1} \sum_{v=n}^{2 n-1} \sum_{k, l=1}^{\infty} \Phi\left(\lambda \mid t_{k m+u, l n+v}-t_{k m+u,(l-1) n+v}-\right. \\
& \left.-t_{(k-1) m+u, l n+v}+t_{(k-1) m+u,(l-1) n+v}\right) \leq \\
& \leq b \Psi\left(\omega_{\varphi}(\lambda x ; r, s)\right) \sum_{u=m}^{2 m-1} \sum_{v=n}^{2 n-1} v_{\Phi}(\lambda x)=b m n \Psi\left(\omega_{\varphi}(\lambda x ; r, s)\right) v_{\Phi}(\lambda x) \text {. }
\end{aligned}
$$


Finally we obtain

$$
\sum_{\mu, \nu=0}^{\infty} \Gamma\left(c \lambda\left|\left(\tau_{m n} x\right)_{k \nu}-(x)_{\mu \nu}\right|\right) \leq b m n \Psi\left(\omega_{\varphi}(\lambda x ; r, s)\right) v_{\Phi}(\lambda x)
$$

for some positive constants $c, b, \lambda$ and for all $m \geq r, n \geq s$, where $r, s$ are nonnegative integers. Hence, taking $m=r$ and $n=s$, we get the inequality $(*)$.

Theorem 2. Let $\Phi, \varphi, \Gamma$ be $\varphi$-functions ( $\Phi$ convex) and let $\Psi$ have the same properties as in the previous theorem. Let $x \in \tilde{x} \in \tilde{X}(\Phi, \Psi)$ and $x \in X_{d}$ (or $\left.x \in X_{i}\right)$. Then $\tau_{r g} x-x \in l^{\Gamma}$ for all $r, s \geq 0$, and $\tau_{r s} x-x \rightarrow 0$ in the sense of modular convergence in $l^{\Gamma}$.

Proof: First, let us remark that the condition $x \in X(\Phi, \Psi)$ implies that $v_{\Phi}(\lambda x)<\infty$ and $r s \Psi\left(\omega_{\varphi}(\lambda x ; r, s)\right)<\varepsilon$ for sufficiently small $\lambda>0$ and for sufficiently large $r$ and $s$, where $\varepsilon$ is an arbitrary positive number. But, an easy computation shows that if the $\varphi$-function $\Phi$ is convex then the conditions $x \in$ $X_{\Phi}$ and $v_{\Phi}(k x)<\infty$ for some positive constant $k$ are equivalent. Applying this observation and Theorem 1 , we conclude that $\tau_{r s} x-x \in l^{\Gamma}$ for all nonnegative integers $r$ and $s$. In order to get the condition $\tau_{r s} x-x \rightarrow 0$ in the sense of modular convergence in $l^{\Gamma}$, it will be necessary to take $r, s \rightarrow \infty$, in the inequality $(*)$.

Theorem 3. Let $x^{p}=\left(t_{\mu \nu}^{p}\right)_{\mu, \nu=0}^{\infty} \in X_{\Phi}, t_{\mu 0}^{p}=t_{0 \nu}^{p}=0$ for $p=1,2, \ldots$ where $\mu, \nu=0,1,2, \ldots$, and let $x^{p}, p=1,2, \ldots$ belong to the $v_{\Phi}$-ball in $X_{\Phi}$, where $\Phi$ is an increasing $\varphi$-function. Then the set of sequences $\left(x^{p}\right)$ is uniformly bounded.

Proof: By assumption $v_{\Phi}\left(k_{0} x^{p}\right) \leq M_{0}$ for $p=1,2, \ldots$, where $k_{0}, M_{0}$ are some positive constants. In consequence, we have

$$
\Phi\left(k_{0}\left|t_{\mu \nu}^{p}\right|\right)=\Phi\left(k_{0}\left|t_{00}^{p}-t_{0 \nu}^{p}-t_{\mu 0}^{p}+t_{\mu \nu}^{p}\right|\right) \leq v_{\Phi}\left(k_{0} x^{p}\right) \leq M_{0} .
$$

Now, applying the properties of $\varphi$-function $\Phi$ we obtain that there exists a positive constant $M$ such that $\left|t_{\mu \nu}^{p}\right| \leq M$ for $\mu, \nu=0,1,2, \ldots$.

Theorem 4. Let $\Gamma, \Phi, \varphi$ be $\varphi$-functions ( $\Phi$ and $\varphi$ are convex) and let $\Psi$ be a nonnegative, nondecreasing function of $u \geq 0$ such that $\Psi(u) \rightarrow 0$ as $u \rightarrow 0+$. Let us suppose that the functions $\Phi, \varphi, \Psi$ and $\Gamma$ satisfy the condiiton 4.2.(i). Moreover, let $\left(x^{p}\right)$ be a sequence such that $t_{\mu 0}^{p}=t_{0 \nu}^{p}=0$ for $\mu, \nu=0,1,2, \ldots, p=1,2, \ldots, \tilde{x}^{p} \in \tilde{x}^{p}, \tilde{x}^{p} \in \tilde{X}(\Phi, \Psi), \tilde{x}^{p} \stackrel{\gamma}{\rightarrow} 0$ as $p \rightarrow \infty$ in $\left\langle\tilde{X}(\Phi, \Psi), \tilde{v}_{\Phi}, \tilde{\rho}_{\varphi}\right\rangle$. Then $\tau_{r s} x^{p}-x^{p} \rightarrow 0$ with respect to modular convergence in $l^{\Gamma}$, as $p \rightarrow \infty$, uniformly for $r \geq 0$ and $s \geq 0$.

Proof: The condition $\tilde{x}^{p} \stackrel{\rightarrow}{\rightarrow} 0$ implies that $\tilde{x}^{p} \in \tilde{K}$, where $\tilde{K}$ is a $\tilde{v}_{\Phi}$-ball, with parameters $k_{0}, M_{0}$, and by Theorem 3 we have $\left|t_{\mu \nu}^{p}\right| \leq M$ for all $\mu, \nu, p$ 
with an $M>0$. Choosing $u_{1}=4 \lambda M, c=a \frac{u_{0}}{u_{1}}$, where $0<\lambda<k_{0}$, and applying the inequality (*), we obtain

(+) $\quad \sum_{\mu, \nu=0}^{\infty} \Gamma\left(c \lambda\left|\left(\tau_{r s} x^{p}\right)_{\mu \nu}-\left(x^{p}\right)_{\mu \nu}\right|\right) \leq b \rho_{\varphi}\left(\lambda x^{p}\right) v_{\Phi}\left(\lambda x^{p}\right) \leq b M_{O} \rho_{\varphi}\left(\lambda x^{p}\right)$.

By assumption there exists a $\lambda>0$ such that for every $\varepsilon>0$ there is an integer $P$ for which

$$
\tilde{\rho}_{\varphi}\left(2 \lambda \tilde{x}^{p}\right)=\inf \left\{\rho_{\varphi}(y): y \in 2 \lambda \tilde{x}^{p}\right\}<\varepsilon
$$

for all $p>P$. In consequence there exist $y^{p} \in 2 \lambda \tilde{x}^{p}$, such that

$$
\rho_{\varphi}\left(y^{p}\right)<\varepsilon \text { for } p>P \text {. }
$$

Since

$$
\rho_{\varphi}\left(\lambda x^{p}\right)=\rho_{\varphi}\left(\frac{y^{p}+\left(2 \lambda x^{p}-y^{p}\right)}{2}\right) \leq \rho_{\varphi}\left(y^{p}\right)+\rho_{\varphi}\left(2\left(\lambda x^{p}-\frac{1}{2} y^{p}\right)\right)
$$

and

$$
\frac{1}{2} y^{p}-\lambda x^{p} \in \bar{c}
$$

then we have

$$
\rho_{\varphi}\left(\lambda x^{p}\right) \leq \rho_{\varphi}\left(y^{p}\right), \text { for } p>P .
$$

By the inequalities $(++)$ and $(++t)$ we obtain

$$
\rho_{\varphi}\left(\lambda x^{p}\right)<\varepsilon
$$

for sufficiently large $p$. Finally, the condition $(t)$ implies that $\tau_{r s} x^{p}-x^{p} \rightarrow 0$ with respect to modular convergence in $l^{\Gamma}$ as $p \rightarrow \infty$, uniformly for $r, s \geq 0$.

\section{References}

1. T.M. JEDRYKA AND J. MUSIELAK, Some remarks on $F$-modular spaces, Functiones et Approximatio 2 (1976), 83-100.

2. J. MusielaK, "Orlicz spaces and modular spaces," Lecture Notes in Math. 1034, Springer-Verlag, Berlin-Heidelberg-New York-Tokyo, 1983.

3. J. Musielak and W. Orlicz, On modular spaces, Studia Math. 18 (1959), 49-65.

4. J. Musielak and A. WaszaK, On two modular spaces, Comment. Math. 23 (1983), 63-70. 
5. J. MUSIELAK AND A. WASZAK, Generalized variation and translation operator in some sequence spaces, Hokkaido Math. Journal 17 (1988), 345-353.

6. A. WASZAK, On convergence in some two-modular spaces, General topology and its relations to modern analysis and algebra, V, Heldermann Verlag Berlin 1982, 667-678.

7. A. WASZAK, On some modular spaces of double sequences I, Commentationes Math. (in print).

Keywords. Sequence spaces, modular spaces

1980 Mathematics subject classifications: $46 \mathrm{~A} 45,46 \mathrm{E} 30$

\author{
Institute of Mathematics \\ Adam Mickiewicz University \\ ui. Matejki $48 / 49$ \\ 60-769 Poznan \\ POLAND
}

Rebut el 16 de Juny de 1989 